\title{
A WEIGHTED NORM INEQUALITY FOR VILENKIN-FOURIER SERIES
}

\author{
JOHN A. GOSSELIN
}

ABSTRACT. Various operators related to the Hardy-Littlewood maximal function have been shown to satisfy a strong type $(p, p)$ condition, $1<p<\infty$, for weighted $L^{p}$ spaces providing the weight function satisfies the $A p$ condition of B. Muckenhoupt. In particular this result for the maximal partial sum operator for trigonometric series was established by $R$. Hunt and W. S. Young. In this note a result similar to that of Hunt and Young is established for Vilenkin-Fourier series, which include Walsh series as a special case.

In a recent paper [5], $R$. Hunt and $W$. Young established that the maximal partial sum operator for the trigonometric system is a bounded operator on the weighted $L^{p}$ spaces, $p>1$, providing the weight function satisfies the $A p$ condition introduced by $\mathrm{B}$. Muckenhoupt [6]. In this note we establish a similar result for the Vilenkin systems which include the Walsh system as a special case. This proof follows closely that in [5] but avoids several technical problems encountered in the trigonometric system due to the discrete nature of the underlying group $G$. In particular, our proof is based on a joint distribution inequality similar to those in [1] and [2].

We assume the reader is familiar with the description and notation of Vilenkin systems $(G, X)$ as discussed in [4]. In particular, we still require that $X$ have a bounded subgroup structure. In the context of Vilenkin systems, a nonne gative weight function $v(x)$ satisfies the $A p$ condition, $p>1$, if there exists a constant $B$ such that

$$
\left(\mu(\omega)^{-1} \int_{\omega} v(x) d \mu(x)\right)\left(\mu(\omega)^{-1} \int_{\omega} v(x)^{-1 /(p-1)} d \mu(x)\right)^{p-1} \leq B
$$

for all cosets $\omega$ of the fundamental sequence of subgroups $\left\{G_{n}\right\}$. Using [3], it is easy to check that each of the following consequences of the $A p$ condition remained valid in the context of Vilenkin systems:

Received by the editors February 25, 1974.

AMS (MOS) subject classifications (1970). Primary 42A56; Secondary 43A15.

Key words and phrases. Weighted norm inequalities, maximal operators, joint distribution inequalities. 
(i) If $v(x)$ satisfies the $A p$ condition with $p>1$, then there exists $r$, $1<r<p$, such that $v(x)$ satisfies the $A_{(p / r)}$ condition.

(ii) Given a measurable set $E$ and a coset $\omega$ with $\mu(E \cap \omega) \leq \epsilon \mu(\omega)$, there exist positive constants $C$ and $\delta$, independent of $E$ and $\omega$, such that $\mu_{\nu}(E \cap \omega) \leq C_{\epsilon}^{\delta} \mu_{\nu}(\omega)$ where $\mu_{\nu}(F)=\int_{F} \nu(x) d \mu(x)$ for any measurable set $F$.

(iii) Let $H f(x)=\sup _{x \in \omega}\left(\mu(\omega)^{-1} \int_{\omega}|f(t)| d \mu(t)\right)$ for any integrable $f$. Then if $f \in L^{p}(G), p>1$, and $v(x)$ satisfies the $A p$ condition, there exists a constant $C p$ independent of $f$ such that

$$
\int_{G} H f(x)^{p} v(x) d \mu(x) \leq C_{p}^{p} \int_{G}|f(x)|^{p} d \mu(x) .
$$

It should be pointed out that the validity of these consequences depends upon the bounded subgroup structure of $X$.

Let $S_{n} f(x)$ denote the $n$th partial sum of the Vilenkin-Fourier series of an integrable $f$, and let $M f(x)=\sup _{n}\left|S_{n} f(x)\right|$. We wish to show that if $v(x)$ satisfies the $A p$ condition, $p>1$, then $f \in L^{p}(G)$ implies

$$
\int_{G} M f(x)^{p} v(x) d \mu(x) \leq C_{p}^{p} \int_{G}|f(x)|^{p} v(x) d \mu(x)
$$

with $C_{p}$ independent of $f$. Following [4] we replace $S_{n} f(x)$ by the modified $n$th partial sum operator

$$
S_{n}^{*} f(x)=\chi_{n}(x) S_{n}\left(f \bar{\chi}_{n}\right)(x)=\left(f * D_{n}^{*}\right)(x),
$$

where $D_{n}^{*}$ denotes the modified $n$th Dirichlet kernel and $*$ denotes convolution over $G$. Setting $M^{*} f(x)=\sup _{n}\left|S_{n}^{*} f(x)\right|$, we will show that

$$
\int_{G}\left(M^{*} f(x)\right)^{p} v(x) d \mu(x) \leq C_{p}^{p} \int_{G}|f(x)|^{p} v(x) d \mu(x) .
$$

For $p>1$, let

$$
H_{p} f(x)=\sup _{x \in \omega}\left(\mu(\omega)^{-1} \int_{\omega}|f(t)|^{p} d \mu(t)\right)^{1 / p}=\left(H_{1}|f|^{p}(x)\right)^{1 / p} .
$$

Following Hunt and Young, we will establish the distributional inequality

$$
\mu_{\nu}\left\{x \in G: M^{*} f(x)>4 \lambda, H_{\nu} f(x) \leq \gamma \lambda\right\} \leq C(\gamma) \mu_{\nu}\left\{x \in G: M^{*} f(x)>\lambda\right\}
$$

for $\gamma \leq \gamma_{0}$, where $C(\gamma) \rightarrow 0$ as $\gamma \rightarrow 0$ and $r$ is given in the first consequence of the $A p$ condition. To see that (2) implies (1), the reader is referred to [5].

To establish (2) we first note that the set $\left\{x \in G: M^{*} f(x)>\lambda\right\}$ is a countable union of disjoint cosets $\omega_{j}$. This follows from the fact that if $m_{k}<$ 
$n \leq m_{k+1}, S_{n}^{*} f(x)$ is constant on cosets of $G_{k+1}$. Furthermore, we may assume each $\omega_{j}$ is maximal in the following sense: If $\omega_{j}=x+G_{k}$, then there exists a point $z_{j} \in \omega_{j}^{*}=x+G_{k-1}$ such that $M^{*} f\left(z_{j}\right) \leq \lambda$. Thus it suffices to prove that for each $\omega_{j}$

$$
\mu_{\nu}\left\{x \in \omega_{j}: M^{*} f(x)>4 \lambda, H_{\gamma} f(x) \leq \gamma \lambda\right\} \leq C(\gamma) \mu_{\nu}\left(\omega_{j}\right)
$$

for $\gamma \leq \gamma_{0^{*}}$. Let $f=f I_{\omega_{j}^{*}}(x)+f I_{G \backslash \omega_{j}^{*}}(x) \equiv f_{1}(x)+f_{2}(x)$ where $I_{E}$ denotes the characteristic function of a measurable set $E$. We recall from [4] that for $p>1$

$$
\int_{G}\left(M^{*} f(x)^{p} d \mu(x) \leq C_{p}^{p} \int_{G}|f(x)|^{p} d \mu(x)\right.
$$

We may assume there exists $u_{j} \in \omega_{j}$ with $H_{r} f\left(u_{j}\right) \leq \gamma \lambda$. Then

$$
\begin{aligned}
\mu\left\{x \in \omega_{j}:\right. & \left.M^{*} f_{1}(x)>\lambda\right\} \leq \lambda^{-r} \int_{G}\left(M^{*} f_{1}(x)\right)^{r} d \mu(x) \\
& \leq C_{r}^{r} \lambda^{-r} \int_{\omega_{j}^{*}}\left|f_{1}(x)\right|^{r} d \mu(x) \leq C_{r}^{r} \lambda^{-r} \mu\left(\omega_{j}^{*}\right)\left(H_{r} f_{1}\left(u_{j}\right)\right)^{r} \leq A C_{r}^{r} \gamma^{r} \mu\left(\omega_{j}\right)
\end{aligned}
$$

since $\mu\left(\omega_{j}^{*}\right) \leq A \mu\left(\omega_{j}\right)$ follows immediately from the assumption on the subgroup structure of $X$. We now show that

$$
\left\{x \in \omega_{j}: M^{*} f(x)>4 \lambda, H_{r} f(x) \leq \gamma \lambda\right\} \subset\left\{x \in \omega_{j}: M^{*} f_{1}(x)>\lambda\right\}
$$

for $\gamma \leq \gamma_{0}$. Recall from [4] that if $n=\Sigma_{s=0}^{\infty} a_{s} m_{s}$,

$$
D_{n}^{*}(x)=\sum_{s=0}^{\infty} D_{m}(x) \chi_{m}^{-a_{s}}\left(\sum_{j=0}^{a_{s}-1} \chi_{m_{s}}^{j}(x)\right) \equiv \sum_{s=0}^{\infty} \Phi_{m_{s}, a_{s}}(x)
$$

where $D_{m_{s}}(x)=m_{s} I_{G_{s}}(x)$. For $x \in \omega_{j}$ and any $n=\Sigma_{s=0}^{\infty} a_{s} m_{s}, s_{n}^{*} f_{2}(x)=$ $s_{n}^{*} f_{2}\left(z_{j}\right)$. To see this let $n_{1}\left(\omega_{j}\right)=\sum_{s=0}^{k-2} a_{s} m_{s}$ and $n_{2}\left(\omega_{j}\right)=n-n_{1}\left(\omega_{j}\right)$. Then

$$
S_{n}^{*} f_{2}(x)=S_{n_{1}\left(\omega_{j}\right)}^{*} f_{2}(x)+S_{n_{2}\left(\omega_{j}\right)}^{*} f_{2}(x)=\left(f_{2} * D_{n_{1}\left(\omega_{j}\right)}^{*}\right)(x)+\left(f_{2} * D_{n_{2}\left(\omega_{j}\right)}^{*}\right)(x) .
$$

Now $f_{2} * D_{n_{2}\left(\omega_{j}\right)}^{*}=0$ since $D_{n_{2}\left(\omega_{j}\right)}^{*}(x)$ has support in $G_{k-1}$. Also $f_{2} *$ $D_{n_{1}\left(\omega_{j}\right)}^{*}$ is constant on cosets of $G_{k-1}$ and in particular on $\omega_{j}$. Thus $S_{n}^{*} f_{2}(x)=S_{n}^{*} f_{2}\left(z_{j}\right)$. Now for $x \in \omega_{j}$ and any $n$, 


$$
\begin{aligned}
\left|S_{n}^{*} f_{2}(x)-s_{n}^{*} f\left(z_{j}\right)\right|= & \left|S_{n}^{*} f_{1}\left(z_{j}\right)\right|=\left|\left(f_{1} * D_{n}^{*}\right)\left(z_{j}\right)\right| \\
= & \left|\int_{\omega_{j}^{*}} f_{1}(t) \sum_{s=0}^{\infty} \Phi_{m_{s}, a_{s}}\left(z_{j}-t\right) d \mu(t)\right| \\
\leq & \left|\int_{\omega_{j}^{*}} f_{1}(t) \sum_{s=0}^{k-2} \Phi_{m_{s}, a_{s}}\left(z_{j}-t\right) d \mu(t)\right| \\
& +\left|\int_{\omega_{j}^{*}} f_{1}(t) \sum_{s=k-1}^{\infty} \Phi_{m_{s}, a_{s}}\left(z_{j}-t\right) d \mu(t)\right| \\
\leq & \int_{\omega_{j}^{*}}|f(t)|\left(\sum_{s=0}^{k-2} a_{s} m_{s}\right) d \mu(t)+\left|s_{n_{2}}^{*}\left(\omega_{j}\right) f\left(z_{j}\right)\right| \\
\leq & m_{k-1} \int_{\omega_{j}^{*}}|f(t)| d \mu(t)+M^{*} f\left(z_{j}\right) \\
\leq & \leq H_{1} f\left(u_{j}^{*}\right)+M_{j}^{*} f\left(z_{j}\right) \leq(\gamma+1) \lambda . \\
& \int_{\omega_{j}^{*}}|f(t)| d \mu(t)+M^{*} f\left(z_{j}\right)
\end{aligned}
$$

It now follows that $M^{*} f_{2}(x) \leq M^{*} f\left(z_{j}\right)+\lambda(\gamma+1) \leq \lambda(\gamma+2)$. Thus for $x \in \omega_{j}$,

$$
M^{*} f(x) \leq M^{*} f_{1}(x)+M^{*} f_{2}(x) \leq M^{*} f_{1}(x)+\lambda(2+\gamma) .
$$

Hence if $M^{*} f(x)>4 \lambda$, it follows that $M^{*} f_{1}(x)>\lambda$ if $\gamma \leq \gamma_{0}<1$. Then for $\gamma \leq \gamma_{0}$

$$
\mu\left\{x \in \omega_{j}: M^{*} f(x)>4 \lambda, H_{r} f(x) \leq \gamma \lambda\right\} \leq \mu\left\{x \in \omega_{j}: M^{*} f_{1}(x)>\lambda\right\} \leq C \gamma^{r} \mu\left(\omega_{j}\right)
$$

by (5). Using the second consequence of the $A p$ condition, we obtain

$$
\mu_{v}\left\{x \in \omega_{j}: M^{*} f(x)>4 \lambda, H_{v} f(x) \leq \gamma \lambda\right\} \leq C\left(\gamma^{r}\right)^{\delta} \mu_{v}\left(\omega_{j}\right) .
$$

Thus (2) is established and the proof is complete.

\section{REFERENCES}

1. D. L. Burkholder, Distribution inequalities for martingales, Ann. of Probo 1 (1973), 19-42. 
2. R. R. Coifman, Distribution function inequalities for singular integrals, Proc. Nat. Acad. Sci. U.S.A. 69 (1972), 2838-2839. MR 46 \#2364.

3. R. R. Coifman and C. Fefferman, Weighted norm inequalities for maximal functions and singular integrals, Studia Math. 51 (no. 3), 241-250.

4. J. A. Gosselin, A. e. convergence of Vilenkin-Fourier series, Trans. Amer. Math. Soc. 185 (1973), 345-370.

5. R. A. Hunt and W. S. Young, A weighted norm inequality for Fourier series, Bull. Amer. Math. Soc. 80 (1974), 274-277.

6. B. Muckenhoupt, Weighted norm inequalities for the Hardy maximal function, Trans. Amer. Math. Soc. 165 (1972), 207-226. MR 45 \#2461.

DEPARTMENT OF MATHEMATICS, SYRACUSE UNIVERSITY, SYRACUSE, NEW YORK 13210

Current address: Department of Mathematics, University of Georgia, Athens, Georgia 30601 